\title{
MECHANISMS OF SEPARATION OF ELEMENTS
}

\author{
GEORGES MICHAUD \\ CERCA, and Département de Physique, Université de Montréal, C. P. 6128, \\ Succursale A, Montréal, H3C 3J7, QUE, CANADA. \\ C. R. Proffitt \\ Space Telescope Science Institute
}

\section{ASTROPHYSICAL CONTEXT}

Since the last IAU Colloquium devoted to Ap stars, the continuous development of high signal to noise observations and of computerized analysis tools have greatly increased the effectiveness of abundance anomalies as a probe of hydrodynamical processes in stars. Until the 1980s, they seemed limited to ApBp and AmFm stars where the effects are largest. More recently the abundances of $\mathrm{Li}$ and $\mathrm{He}$ have been suggested to play important diagnostic roles for hydrodynamics in both the Sun (Proffitt and Michaud 1991b, Pinsonneault et al. 1989) and Halo stars (Proffitt and Michaud 1991a, Pinsonneault et al. 1992, Vauclair 1988, Charbonnel et al. 1992, Deliyannis and Demarque 1991). The observation of a $\mathrm{Li}$ abundance gap in $\mathrm{F}$ stars of clusters has raised the question of the link between these objects and the AmFm stars (Michaud 1986, Charbonneau and Michaud 1988, 1991) or alternately with solar type stars. The observed $\mathrm{Li}$ abundance on the subgiant branch sheds some light on this problem (Charbonneau et al. 1989; Charbonnel and Vauclair 1992). In solar type stars, the observed $\mathrm{Li}$ abundance constrains the superficial turbulence that may inhibit surface He gravitational settling (Proffitt and Michaud 1991b) whose effects can be detected using solar seismology (Guzik and Cox 1992, Dziembowski $e t$ al. 1992).

Research on peculiar stars consequently acquires a new dimension. These objects cease being freaks outside of the main stream of astrophysical research. Instead, they open the way to an understanding of the competition between hydrodynamical and particle transport processes that play an important role in most stars (see Michaud 1991a and most papers in that Symposium proceeding).

In past reviews (Michaud 1976, 1980, 1986) the emphasis was put on observational evidence supporting the importance of atomic diffusion as a particle transport process. Here instead, we first review recent developments in the calculations of particle transport processes (\$II). The effect of atomic diffusion on stellar evolution for the Sun, as well as in F and A stars will be briefly mentioned (§III) as well as developments concerning physical processes in the presence of magnetic fields ( $\$ I V ;$ see also Babel, this volume), followed by recent results concerning the competition between atomic diffusion, turbulence and mass loss $(\S \mathrm{V}$; see also Charbonneau, this volume, for a discussion of accretion, mass loss and meridional circulation) in particular in relation to $\mathrm{Li}$ abundance observations. 


\section{PARTICLE TRANSPORT PROCESSES}

Various formulations of the diffusion equations and approximations for the diffusion coefficients have been used over the last few years. They are reviewed in more detail in Michaud and Proffitt (1992). We here only briefly mention the main points in a). A more detailed discussion of light induced drift is presented in b) since it may well play a significant role in peculiar stars.

\section{a) Diffusion Equations and Coefficients}

The various formulations of the diffusion velocity equations (see, for instance, Chapman and Cowling 1970 and Burgers 1969) can all be made to give essentially the same results. The results do depend however on the diffusion coefficients. Approximations have been made by many authors in order to avoid carrying out triple integrals numerically in the evaluation of the coefficients. This leads to approximate analytic solutions which are accurate in the limit of very dilute plasmas. The collision integrals are then proportional to

$\ln \Lambda_{i j}=\ln \left(\right.$ constant $\left.\frac{2 k T \lambda_{d}}{z_{i} z_{j} e^{2}}\right)$,

often referred to as the Coulomb logarithm. For very dilute plasmas, $\ln \Lambda_{\mathrm{ij}}$ is large and its value does not depend sensitively on the value of the constant appearing in the logarithmic term. This constant depends on approximations made in arriving at an analytic expression. In the interior of the sun and halo stars, $\ln \Lambda_{i j}$ is not much larger than one (see Fig. 1 and 2 of Michaud and Proffitt 1992). The use of analytic expressions is then unjustified. One should instead use the results of a complete numerical integration for the collision coefficients (Paquette et al. 1986). Michaud and Proffitt (1992) have proposed analytic expressions that they calibrated to the results of Paquette $e t$ al. in order to facilitate their use in evolutionary calculations. They are more accurate and as easy to use as the expressions proposed by Bahcall and Loeb (1990).

There remain uncertainties in the calculations of the collision integrals. They come from the physical assumptions made in arriving at the collision integrals and diffusion equations, not from the mathematical simplifications needed to derive simple analytic expressions (see Michaud 1991 for a discussion). In main sequence stars, the uncertainties are probably larger for highly ionized heavy elements ( $Z$ large) than for $\mathrm{H}$ or $\mathrm{He}$.

The abundance variations that diffusion leads to depend on the relative value of radiative acceleration and gravity. The use of atomic data banks accumulated by the Opacity project and by Kurucz (1991) should allow to reduce the uncertainty in the value of $g_{R}$ from a factor of 2-3 to a factor of 1.2-1.4. Using the results of the Opacity project Alecian, Michaud and Tully (1992) have obtained accurate $\mathrm{g}_{\mathrm{R}}$ for $\mathrm{Fe}$. They have shown that $\mathrm{Fe}$ can be supported over a large fraction of the envelope and since $\mathrm{Fe}$ has been found to be the dominant opacity source over a certain $\mathrm{T}$ range in stellar envelopes (Rogers and Iglesias 1992), the local overabundances of Fe created by atomic diffusion could have significant impact on stellar evolution models. One should calculate the atomic diffusion of $\mathrm{Fe}$ at the same time as one carries out the stellar evolution calculations. Local overabundances of Fe could lead to a kappa mechanism to drive the observed pulsations (see Kurtz this volume) of Ap stars (see also Cox et al. 1992 for a similar suggestion in a different context). 
Similar effects may be expected from $\mathrm{C}, \mathrm{N}$ and $\mathrm{O}$ and probably some other $\mathrm{Fe}$ peak elements.

\section{b) Light Induced Drift}

Light induced drift (LID) is a physical phenomenom observed in certain laboratory experiments (Atutov 1986). It is different from the effect of $g_{R}$ in driving diffusion. It does not arise from the transfer of momentum from the radiation field to atoms but from the transfer of momentum from one type of atoms to another through selective excitation of one type of atoms.

LID arises when the radiation field is anisotropic within a line profile. The simplest case is that of a purely Doppler broadened resonance line (from the ground state of the neutral atom) of a trace element. When the atom is moving away from the star it sees the flux (more precisely, the anisotropic part of the intensity) on the blue side of the line centre in the star reference system. Assume that the flux is larger on the blue side. More atoms are excited in the blue half of the profile (when they move away from the star) than in the red half (when they move toward the star).

The collisional cross section (for momentum exchange with the main gas) of the excited state is generally larger than that of the ground state. Consider neutral $\mathrm{He}$ colliding with neutral $\mathrm{H}$ in its ground state. The collision cross section is mainly determined by the radius of the electrons around the He nucleus and, in the $\mathrm{He}$ ground state, the electrons are much more tightly bound than in the excited state. The collision rate is about nine times larger for the excited state of $\mathrm{He}$ with $\mathrm{H}$ than for the ground state of $\mathrm{He}$. In the direction which has most excited atoms there will be a larger collision rate or, equivalently, a smaller mean free path. This imbalance induces a drift of the element in the direction with least excited atoms (toward the centre in this case).

The preceding discussion has been made in terms of the frequency anisotropy of the radiative flux. It can easily be seen from symmetry arguments that only the spatially anisotropic part of the radiative intensity is involved since otherwise atoms would have no way to know in which direction they should drift. It is then only necessary to consider the part of the radiation field that is spatially inhomogeneous. This could involve all the odd moments of the radiation field.

LID was suggested by Atutov and Shalagin (1988) to be more efficient than radiation driven diffusion in causing particle transport in stars. LeBlanc and Michaud (1992) did detailed calculations for a few elements in model atmospheres of A stars. They compare velocities induced by LID and by $g_{R}$ for a given line. They obtained, when the diffusion approximation may be used for the radiative flux:

$$
\begin{aligned}
& \frac{\mathrm{V}_{\mathrm{LID}}}{\mathrm{V}_{\mathrm{RAD}}}=610^{10}\left[\frac{\mu_{1}-\mu_{0}}{\mu_{0}}\right]\left[\frac{\mathrm{A}_{10}}{\mathrm{~A}_{10}+\mu_{1}}\right] \frac{\mathrm{g}_{1}}{\mathrm{~g}_{0}}\left[\frac{\mathrm{V}_{\mathrm{T}}}{\mathrm{T}^{2} \mathrm{u}^{3} \mathrm{f} \mathrm{D}}\right] \mathrm{W}, \\
& \mathrm{W}=\frac{\int_{0}^{\nu} \frac{\mathrm{K}_{\nu}(\mathrm{A})}{\mathrm{K}_{\nu}} \mathrm{d} \nu-\int_{\nu_{0}}^{\infty} \frac{\mathrm{K}_{\nu}(\mathrm{A})}{\mathrm{K}_{\nu}} \mathrm{d} \nu}{\int_{0}^{\infty} \frac{\mathrm{K}_{\nu}(\mathrm{A})}{\mathrm{K}_{\nu}} \mathrm{d} \nu},
\end{aligned}
$$

where $A$ is the atomic number of the element, $f$ is the oscillator strength of the transition considered and $K \nu(A)$ is the related opacity, $g_{0}$ and $g_{1}$ are the statistical weights of the lower and upper energy levels, $u=h \nu_{0} /(k T)$ and $D$ is the diffusion 
coefficient. The quantity $\mathrm{W}$ is a measure of the anisotropy of the flux within the line. It appears in the ratio of the two velocities since the radiative acceleration is caused by all photons within the line whereas LID comes from a substraction of the excitation caused on each side of a Doppler broadened line. The collisional frequencies $\mu_{0}$ and $\mu_{1}$ are the momentum transfer frequencies for the ground and first excited states.

The potential importance of LID may be estimated by considering a fictitious element with a first excited level at $7 \mathrm{eV}$ whose statistical weight equals that of the ground state $\left(g_{1}=g_{0}\right)$. Assume a thermal velocity $V_{T}=10^{6} \mathrm{~cm} \mathrm{~s}^{-1}$ and a resonance line with $f=1$. We maximize the effect by assuming that the element is neutral so that we may use $\left(\mu_{1}-\mu_{0}\right) / \mu_{0}=1$ for the ratio of the difference of the collisional rates. The diffusion coefficient, $D$, is calculated for neutral $\mathrm{He}$ (when $N_{\mathrm{p}}=10^{16} \mathrm{~cm}^{-3}$ ) in protons. The anisotropy, $\mathrm{W}$, is assumed equal to 1 to maximize the effect. Then one obtains $V_{D} / V_{R A D} \sim 210^{2}$, so that drift dominates over radiation driven diffusion.

Under favourable conditions, LeBlanc and Michaud then confirm Atutov and Shalagin (1988)'s result that LID dominates particle transport. The question is then to what extent do the assumed ideal conditions materialize. In stellar atmospheres, most elements are either ionized or there is a hydrogen convection zone efficiently mixing all elements. Consider an $\mathrm{HgMn}$ star of $12000 \mathrm{~K}$; all elements except $\mathrm{He}$ and $\mathrm{Ne}$ are ionized. Since they are colliding mainly with protons, the momentum exchange rate is mediated by the Coulomb interaction which is not expected to be strongly affected by the excitation of electrons in the core. Using approximations that would need to be confirmed by more detailed atomic physics calculations, LeBlanc and Michaud (1992; §III) obtain $\left(\mu_{1}-\mu_{0}\right) / \mu_{0}=10^{-5}$ to $10^{-4}$ in this case. The diffusion coefficient is however also reduced by a factor of about 100. An anisotropy $\mathrm{W}=1$ is unrealistic except for isotopes; taking $\mathrm{W}=10^{-2}$ to $10^{-1}$ appears more realistic and leads (taking all corrections into account and assuming everything thing else to remain the same) radiation driven diffusion to dominate by a factor of 10 to 100 over LID. For Li in F stars, they obtain that radiation driven diffusion dominates by a factor of 100 .

In the previous discussion, only one line was considered. For most elements, the anisotropy is caused by the random presence of a neighbouring line. This occurs as frequently on the blue as on the red side of the line and the effect of LID then cancels on a random basis. This reduces the relative effect of LID by a factor of 10 when many lines (of order 100) are involved in the radiative acceleration calculations. For most elements in most stars (outside of convection zones), the effect of LID should then be a factor of about 100 smaller than radiation driven diffusion.

The maximum relative effect of LID is expected for an element such as $\mathrm{He}$ where all the lines of the dominant isotope $\left({ }^{4} \mathrm{He}\right)$ cause a frequency anisotropy on the same side of the lines of the less abundant isotope and when He is substantially neutral. On Figure 4 of LeBlanc and Michaud (1992), is shown a comparison of $\mathrm{LID}$ and radiation driven diffusion in an atmosphere of $T_{\text {eff }}=18000 \mathrm{~K}, \log \mathrm{g}=4$ and $N(\mathrm{He}) / \mathrm{N}(\mathrm{H})=0.1$. The effect of LID is measured by the acceleration, $\mathrm{g}_{\mathrm{LD}}$, that would be needed in the diffusion equation to lead to the LID velocity. The effect of LID dominates, for $\mathrm{N}(\mathrm{He}) / \mathrm{N}(\mathrm{H})=0.1$ and $1 \%{ }^{3} \mathrm{He}$, over the interval $0<$ $\log \tau_{\mathrm{R}}<1.8$. The effect of LID was overestimated in these calculations by a factor of 2 to 4 and the interval over which LID dominates is more appropriately $0.5<\log$ $\tau_{\mathrm{R}}<1.5$. This is still an important interval. There is then at least one case where LID is expected to dominate particle transport in stars. As the He abundance is reduced, the effect of LID progressively decreases. It becomes negligible once the 
evolutionary calculations of $A$ and $F$ stars.

The stellar evolution was calculated for models with mass varying from 1.2 to $2.2 \mathrm{M}_{\odot}$ to cover the $\mathrm{T}_{\text {eff }}$ range of the $\mathrm{Li}$ gap and the Am-Fm stars (Richer 1992). The OPAL opacities (Rogers and Iglesias 1992) were used.

Consider, for example, a model with $T_{\text {off }}=7800 \mathrm{~K}$ on the zero age main sequence; its $\mathrm{T}_{\text {eff }}$ goes down to $6300 \mathrm{~K}$ just before crossing to the giant branch ( see Fig. 1 of Michaud and Richer 1992). The time evolution of the $\mathrm{Li}$ abundance is governed locally by the relative values of the radiative acceleration on $\mathrm{Li}\left(\mathrm{g}_{\mathrm{R}}(\mathrm{Li})\right)$ and of gravity. On arrival on the main sequence, $\mathrm{Li}$ is not supported below the convection zone since it is mainly in the He like configuration. Slightly above $\Delta \mathrm{M} / \mathrm{M}_{\odot}=10^{-8}$ and down to $\Delta \mathrm{M} / \mathrm{M}=10^{-6}, \mathrm{~g}_{\mathrm{R}}(\mathrm{Li})>\mathrm{g}$ and $\mathrm{Li}$ is pushed to $\Delta \mathrm{M} / \mathrm{M}_{\odot}$ $=10^{-8}$ where a $\mathrm{Li}$ cloud forms. As time passes the bottom of the convection zone approaches the $\mathrm{Li}$ cloud that has formed during evolution where $\mathrm{g}_{\mathrm{R}}(\mathrm{Li})=\mathrm{g}$.

By comparing these calculations, done entirely from first principles, to observations of $\mathrm{Li}$ in clusters (Richer and Michaud 1992), it is found that: a) the position of the $\mathrm{Li}$ gap is at $\mathrm{T}_{\text {eff }}=6740 \mathrm{~K}$, in agreement with observations; b) the depth of the $\mathrm{Li}$ gap is by a factor of 100 at the age of the Hyades using the OPAL tables, in agreement with observations ; c) the width of the gap is only half as wide as observed; d) as clusters become older the position of the gap shifts to slightly lower $\left.T_{\text {eff }} ; e\right)$ for stars with $T_{\text {eff }}>7000 \mathrm{~K}$, there is a need for an alternate hydrodynamical process competing with diffusion. This is the AmFm domain where there is a need for such a process to reduce the anomalies that uninhibited diffusion leads to (see also Alecian, this volume).

A similar time dependence of the abundance is to be expected for other elements in AmFm stars. Calculations are in progress for Be, C, N, O and Fe. For heavy elements, the cloud is expected when there is a state of ionization where $g_{R}<$ g. This state has to dominate $g_{R}$ so that others do not fill the gap. This may not always occur. Only detailed calculations will tell which elements are similarly affected. A comparison to observations of a large number of heavy elements in AmFm stars of clusters should allow a rigorous test of a model for these objects. Because of the potential presence of clouds of different elements, detailed evolutionary calculations are needed.

\section{ATMOSPHERES OF MAGNETIC STARS}

In the presence of a strong horizontal magnetic field, protons and electrons are frozen in, while neutral hydrogen can diffuse vertically. As a consequence, the hydrostatic equilibrium density gradient applies to neutral hydrogen alone and, coupled with the progressive ionization of hydrogen in a $9000 \mathrm{~K}$ model atmosphere, this leads to a much compressed atmospheric region (see Babel and Michaud 1991). More detailed modelling is in progress (LeBlanc Michaud and Babel, in preparation). In particular, it is important to determine if hydrogen lines are significantly affected. Since this is calculated from first principles but leads to large effects only if the horizontal magnetic field suppresses convection, this may lead to a diagnostic of the effect of horizontal magnetic fields on convection.

Babel (this volume) discusses many developments in the modelling of magnetic stars. 
total $\mathrm{He}$ abundance has been reduced to $10^{-5}$ of it original value. This is simply understood as the consequence of the disappearance of the lines causing the flux anisotropy. The effect of LID is important only for the less abundant isotope.

Assume that a star, such as 3 Cen $\mathrm{A}$, starts its main sequence life with normal abundances of the two He isotopes. Assume that its outer regions are stable. Particle diffusion leads to the progressive decrease of the abundances of both ${ }^{3} \mathrm{He}$ and ${ }^{4} \mathrm{He}$ throughout the atmosphere and to the progressive replacement of ${ }^{4} \mathrm{He}$ by ${ }^{3} \mathrm{He}$ starting only after underabundances by factors of 10 to 100 have materialized. This leads to approximately the observed ${ }^{3} \mathrm{He} /{ }^{4} \mathrm{He}$ ratio only after time scales 3 times longer than the evolutionary time scale for this star (Martel 1979; Michaud $e t$ al. 1979; Vauclair Michaud and Charland 1973). This difficulty would be alleviated by LID. Since the upwards drift of ${ }^{3} \mathrm{He}$ would start, over the interval $0.5<\log \tau_{R}<$ 1.5 , immediately after the star formed, the time needed for ${ }^{3} \mathrm{He}$ to replace ${ }^{4} \mathrm{He}$ should be reduced by a factor of 3-10. This remains to be confirmed by detailed time dependent calculations including the effect of LID and without the overestimates in the evaluation of LID that were made by LeBlanc and Michaud. This involves calculating triple integrals.

No other evident candidate was identified where LID may play a dominant role. LeBlanc and Michaud concluded that LID was unlikely to play a dominant role for the isotope separation of $\mathrm{Hg}$ because $\mathrm{Hg}$ is too ionized. This should be checked when detailed diffusion calculations are made for that element.

The effect of LID should further be checked in models for cool magnetic stars where the convection zones may be suppressed by the magnetic field (see Babel, this volume) and where more chemical elements are largely neutral. It would be largest for elements with relatively large first ionization potentials.

\section{THE SUN, F AND A STARS}

The effect of atomic diffusion on the evolution of the Sun and of F and A stars has been reviewed in more detail in Michaud and Proffitt (1992) and in Michaud and Richer (1992). Only the main points are mentioned here.

For the Sun, disagreements among previous studies have been analyzed by Proffitt and Michaud (1991b). If diffusion is not inhibited below the convection zone, the current surface mass fraction helium abundance of the Sun, $Y_{a}$, should be about $0.03(10 \%)$ lower than the initial solar helium abundance.

The current solar $\mathrm{Li}$ abundance is 100-200 times smaller than the initial value and it appears difficult to account for all of the depletion by pre-main sequence burning. Some mixing appears needed to carry the Li to a temperature where it burns. While the cause and nature of main sequence mixing is controversial, observational data can constrain its magnitude. Figures 1 to 3 of Proffitt and Michaud (1991b) show that it does not seem possible to reduce the surface settling by more than a factor of two without over-depleting $\mathrm{Li}$.

Observations of solar p-modes provide a means for directly measuring the $\mathrm{He}$ abundance of the surface convection zone (see the discussion in Dziembowski $e t$ al. 1992 and in Kosovichev et al. 1992). These measurements suggest a surface $\mathrm{He}$ abundance consistent with the diffusion predictions noted above (see also Guzik \& Cox 1992). Once current uncertainties in other physics (opacities, abundances, and convection theory) are resolved, the oscillations should provide a direct test of turbulent mixing in $\mathrm{G}$ dwarfs.

Using the Sun, the value of $\alpha$, the ratio of the mixing length to the pressure scale height, was calibrated, so that no arbitrary parameter remained in the 
evolutionary calculations of $\mathrm{A}$ and $\mathrm{F}$ stars.

The stellar evolution was calculated for models with mass varying from 1.2 to $2.2 \mathrm{M}_{\odot}$ to cover the $\mathrm{T}_{\text {eff }}$ range of the $\mathrm{Li}$ gap and the Am-Fm stars (Richer 1992). The OPAL opacities (Rogers and Iglesias 1992) were used.

Consider, for example, a model with $\mathrm{T}_{\text {eff }}=7800 \mathrm{~K}$ on the zero age main sequence; its $T_{\text {eff }}$ goes down to $6300 \mathrm{~K}$ just before crossing to the giant branch ( see Fig. 1 of Michaud and Richer 1992). The time evolution of the $\mathrm{Li}$ abundance is governed locally by the relative values of the radiative acceleration on $\mathrm{Li}\left(\mathrm{g}_{\mathrm{R}}(\mathrm{Li})\right)$ and of gravity. On arrival on the main sequence, $\mathrm{Li}$ is not supported below the convection zone since it is mainly in the He like configuration. Slightly above $\Delta \mathrm{M} / \mathrm{M}_{\odot}=10^{-8}$ and down to $\Delta \mathrm{M} / \mathrm{M}=10^{-6}, \mathrm{~g}_{\mathrm{R}}(\mathrm{Li})>\mathrm{g}$ and $\mathrm{Li}$ is pushed to $\Delta \mathrm{M} / \mathrm{M}_{\odot}$ $=10^{-8}$ where a $\mathrm{Li}$ cloud forms. As time passes the bottom of the convection zone approaches the $\mathrm{Li}$ cloud that has formed during evolution where $\mathrm{g}_{\mathrm{R}}(\mathrm{Li})=\mathrm{g}$.

By comparing these calculations, done entirely from first principles, to observations of $\mathrm{Li}$ in clusters (Richer and Michaud 1992), it is found that: a) the position of the Li gap is at $T_{\text {eff }}=6740 \mathrm{~K}$, in agreement with observations; b) the depth of the Li gap is by a factor of 100 at the age of the Hyades using the OPAL tables, in agreement with observations ; c) the width of the gap is only half as wide as observed; d) as clusters become older the position of the gap shifts to slightly lower $\left.T_{\text {eff }} ; e\right)$ for stars with $T_{\text {eff }}>7000 \mathrm{~K}$, there is a need for an alternate hydrodynamical process competing with diffusion. This is the AmFm domain where there is a need for such a process to reduce the anomalies that uninhibited diffusion leads to (see also Alecian, this volume).

A similar time dependence of the abundance is to be expected for other elements in AmFm stars. Calculations are in progress for Be, C, N, O and Fe. For heavy elements, the cloud is expected when there is a state of ionization where $g_{R}<$ g. This state has to dominate $g_{R}$ so that others do not fill the gap. This may not always occur. Only detailed calculations will tell which elements are similarly affected. A comparison to observations of a large number of heavy elements in AmFm stars of clusters should allow a rigorous test of a model for these objects. Because of the potential presence of clouds of different elements, detailed evolutionary calculations are needed.

\section{ATMOSPHERES OF MAGNETIC STARS}

In the presence of a strong horizontal magnetic field, protons and electrons are frozen in, while neutral hydrogen can diffuse vertically. As a consequence, the hydrostatic equilibrium density gradient applies to neutral hydrogen alone and, coupled with the progressive ionization of hydrogen in a $9000 \mathrm{~K}$ model atmosphere, this leads to a much compressed atmospheric region (see Babel and Michaud 1991). More detailed modelling is in progress (LeBlanc Michaud and Babel, in preparation). In particular, it is important to determine if hydrogen lines are significantly affected. Since this is calculated from first principles but leads to large effects only if the horizontal magnetic field suppresses convection, this may lead to a diagnostic of the effect of horizontal magnetic fields on convection.

Babel (this volume) discusses many developments in the modelling of magnetic stars. 


\section{TURBULENCE AND MASS LOSS}

In the preceding sections, all results were obtained from first principles. In this section, we give examples of how two processes, turbulence and mass loss, that currently require arbitrary parameters for their description, might affect the abundance anomalies of light elements.

The extent to which material mixing occurs in radiatively stable regions of stellar interiors is one of the major unknowns in the physics of main-sequence stars. While the abundance anomalies of many elements are probably affected by turbulence, those of the light elements are specially affected since they are destroyed, close to the surface, by nuclear reactions. Observations of $\mathrm{Li}$ and the other light elements offer direct clues about mixing in outer stellar layers. Many authors have suggested that there is a close relationship between material mixing and the transport of angular momentum, although Garcia Lopez and Spruit (1991) have suggested that turbulence generated by g-mode oscillations might also be relevant. Garcia Lopez and Spruit can reproduce the F-star Li gap only if they multiply their estimate of turbulence by an arbitrary factor of 15 .

One especially challenging observational constraint on rotationally induced $\mathrm{Li}$ depletion mechanisms in low mass stars, is the observation that, in the Pleiades and $\alpha$ Per, the fastest rotating, most active stars tend to have the highest $\mathrm{Li}$ abundances (see Soderblom et al. 1992 for a discussion of these relations in the Pleiades). This can apparently not be explained by an age spread. As current rotational mixing theories result in more mixing at higher rotation rates, something is being left out of the theory of pre-main-sequence Li depletion.

Schatzman (1977) made the earliest attempts to constrain the scale of turbulent mixing in the solar interior by using the observed solar Li depletion, and by assuming that the differential rotation in radiative regions was maintained in a state of marginal stability. He assumed that this resulted in a turbulent diffusion coefficient proportional to, but much larger than, microscopic viscosity. While it is now apparent that this simple formulation is inadequate, it did establish the scale of turbulence needed to explain the observed solar $\mathrm{Li}$ depletion.

Most recent discussions of rotationally induced mixing, assume that the turbulent mixing relevant for Li burning is generated either by the shear instability resulting from differential rotation (Spiegel \& Zahn 1970, Zahn 1974), or by instabilities related to meridional circulation (e.g. Chaboyer \& Zahn 1992). Other instabilities may dominate the production of turbulence in the deep stellar interior, but these will not be discussed here.

Low mass stars arrive on the ZAMS with high rotational velocities and spin down by angular momentum loss via a magnetic wind (Schatzman 1958, Weber \& Davis 1967). Mixing due to shear instabilities in the differential rotation from this magnetic braking of the surface rotation has been suggested to explain the slow destruction of Li that apparently affects the Sun and lower mass Pop. I stars. Detailed stellar evolutionary models that follow the interior angular momentum and chemical distribution as a function of time have been presented by Pinsonneault $e t$ al. $(1989,1990)$ using the Yale Rotating Stellar Evolution Code (YREC). They use estimates for the onset of various hydrodynamic instabilities, and assume solid body rotation and efficient chemical mixing along equipotential surfaces. They also assume that both angular momentum and chemical transport can be modeled by a 1-D diffusion equation, with the value for the turbulent diffusion coefficient for chemical species being proportional to that for angular momentum. They conclude that the transport in the outer layers of solar type stars is dominated by the secular shear 
instability and that the transport of material needs to be $\sim 30$ times less efficient than that of angular momentum in order to match both the observed solar rotation and $\mathrm{Li}$ depletion.

While the YREC models can qualitatively reproduce the observed $\mathrm{Li}$ abundance as a function of time for stars of about one solar mass, there are a number of improvements needed. For example, they assume that the angular momentum loss rate is proportional to $\Omega^{3}$ at all angular velocities. It has recently become apparent that this overestimates the angular momentum loss at very high rotation rates (see the discussion in Charbonneau 1992). The details of the assumed internal angular velocity distributions of the current YREC ZAMS models and their subsequent rotational evolution are thus rather questionable. It will be interesting to see how a more realistic angular momentum loss law will affect their detailed results. In addition, the YREC models offer no apparent explanation of the observed positive correlation between high rotation and high $\mathrm{Li}$ abundance observed in the Pleiades and $\alpha$ Per.

For higher mass stars, such as those in the Hyades Li gap, even greater uncertainties exist. Rotational velocities increase with increasing $\mathrm{T}_{\text {eff }}$ in the Hyades Li gap. This appears to be due to the decreasing efficiency of angular momentum loss as the convective zone thickness decreases, but there are no good quantitative description. For these objects, it is more difficult to disentangle the relative contributions of surface angular momentum loss and angular momentum transport from the interior to the observed rotational velocity evolution than for lower mass stars.

The first YREC models (Pinsonneault et al. 1990) did not reproduce simultaneously the observed rotation and $\mathrm{Li}$ abundances in the $\mathrm{F}$ star Hyades gap. Better fits have been obtained by approaches that assume that the F star Li gap is the result of turbulence related to meridional circulation, as this depends more on a given star's current rotational velocity, and less on the amount of differential rotation than is the case for the secular shear instability. The exact form of the meridional flow, and its relation to the generation of turbulence remains controversial (Charbonneau, this volume).

Chaboyer \& Zahn (1992) and Zahn (1992) assume that turbulence is highly anisotropic and much larger in the horizontal than in the vertical direction. They conclude that this leads to solid body rotation and constant chemical abundances on equipotential surfaces. The vertical transport of material can then be modeled by a 1-D pure diffusion equation, while the transport of angular momentum requires a more complex 1-D equation that includes an advective term. Charbonneau (1992) finds that detailed 2-D calculations support this picture if the horizontal turbulence is arbitrarily assumed to be very strong. Zahn (1992) estimates that the interaction between differential rotation caused by surface angular momentum loss and meridional circulation leads to substantial turbulent mixing, while the same stellar rotation rate in the absence of angular momentum loss results in much reduced chemical mixing. This can potentially explain both the hot and cool sides of the $\mathrm{Li}$ gap, and also allow turbulence to be sufficiently small in A and B stars rotating at $<100 \mathrm{~km} \mathrm{~s}^{-1}$ that sufficient gravitational settling can occur to allow the observed diffusive abundance anomalies. However, the detailed calculations needed to support this picture have yet to be done, and it remains to be seen if Zahn's approach can explain the observed abundance and rotational velocity patterns without the necessity of arbitrarily fine tuning the theory's parameters.

Charbonnel, Vauclair \& Zahn (1992) and Charbonnel \& Vauclair (1992) have undertaken calculations based on Zahn's ideas. They have however, assumed solid 
body rotation and taken a fixed form for the velocity field of the meridional circulation. While they can, with appropriate adjustment of the overall scale of the turbulence, fit the cool side of the Li gap well, they must make additional assumptions about the nature of turbulence to explain the hot side of the $\mathrm{Li}$ gap. Their formulation also clearly predicts too much turbulence in AmFm and HgMn stars to allow sufficient gravitational settling to explain the observed patterns of abundance anomalies. This suggests that they are overestimating the amount of turbulent mixing caused by meridional circulation in stars that rotate as solid bodies.

While the narrow dispersion of $\mathrm{Li}$ abundances in halo dwarfs with $\mathrm{T}_{\text {eff }}>$ 5500 K (Spite \& Spite 1982, and Deliyannis, Pinsonneault, \& Duncan 1992) suggests that there has been little depletion since these stars formed, alternative interpretations have been suggested. Recent calculations with the YREC (Pinsonneault, Deliyannis and Demarque 1992) have led to a uniform depletion by a factor of five to ten of the surface Li of halo stars. This depends, in particular, on the range of rotational velocities assumed for halo stars at their formation. The Pinsonneault et al. results help to constrain the circumstances under which a large uniform depletion is possible.

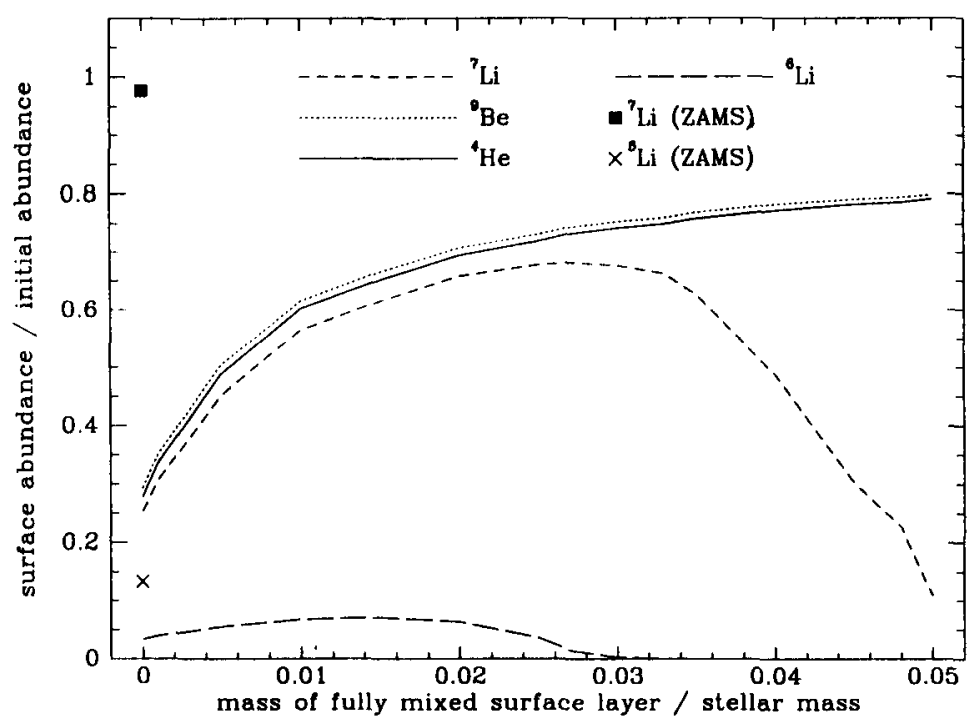

Figure 1 Fractional reduction (at $15 \mathrm{Gyr}$ ) of the surface abundance of a few elements as a function of the stellar mass assumed mixed. See the text.

Indeed, when gravitational settling is taken into account, the minimum possible ${ }^{7} \mathrm{Li}$ depletion in $15 \mathrm{Gyr}$ old metal poor dwarfs is far from negligible, regardless of the amount of internal mixing. In figure 1 we show the results for the surface abundances at $15 \mathrm{Gyr}$ in a $0.75 \mathrm{M}_{\odot}$ star with $\log \mathrm{Z} / \mathrm{Z}_{\odot}=-2.3$, if it is arbitrarily assumed that a surface layer of a given mass is forced to be completely mixed throughout the star's evolution. The thicker the mixed surface layer, the smaller the 
surface abundance decrease due to gravitational settling, but mixing more than the outer $3 \%$ of the mass begins to increase the nuclear destruction of ${ }^{7} \mathrm{Li}$. As a result, at this age, the maximum surface abundance of ${ }^{7} \mathrm{Li}$ is at most $68 \%$ of its initial value. No distribution of internal mixing can give less depletion. Also, the lack of an observed Pop. II $\mathrm{Li}$ dip near $6200 \mathrm{~K}$ (if confirmed), suggests that at least the outer $1 \%$ of the mass (but less than $3 \%$ ) in stars near this $T_{\text {eff }}$ is well mixed, even though the surface convection zone contains only $510^{-4}$ of the stellar mass. Theoretical estimates of the original $\mathrm{Li}$ abundance of Pop. II dwarfs will become truly reliable only after Li depletion in Pop. I dwarfs is more completely understood.

While Richer (1992) concludes that atomic diffusion alone cannot explain all $\mathrm{Li}$ abundances for $\mathrm{T}_{\text {eff }}>7000 \mathrm{~K}$ in the Hyades, the relative importance of turbulence, atomic diffusion and mass loss is not yet established. Results presented by Michaud and Richer (1992) show that the $\mathrm{Li}$ clouds that form within stars are affected by mass loss as soon as the mass loss rate exceeds a critical value:

$\frac{\mathrm{dM}}{\mathrm{dt}}=\frac{1.610^{-15} \mathrm{M} \mathrm{A} \mathrm{T}_{5}^{1.5}}{\mathrm{z}^{2}}$,

where $M$ is in solar mass, $A$ is the atomic mass number and $Z$ the charge of the ion considered. The critical rates are, in F stars, between $10^{-16}$ and $10^{-13} \mathrm{M}_{\odot} \mathrm{yr}^{-1}$. Since the measured solar mass loss rate is $10^{-14} \mathrm{M}_{\odot} \mathrm{yr}^{-1}$ and $\mathrm{F}$ stars have smaller convection zones than the Sun, it is plausible that they have a mass loss rate within the range of the critical mass loss rate. Such mass loss rates were modeled by Richer (1992) and some results reviewed by Michaud and Richer (1992). The modelling of particle transport for more elements in AmFm stars should allow to determine the relative role of atomic diffusion, mass loss and turbulence in AmFm stars and in the Li gap.

\section{REFERENCES}

Alecian, G., Michaud, G., and Tully, J. 1992, preprint.

Atukov, S. N. 1986, Phys. Lett. A, 119, 121.

Atukov, S. N., and Shalagin, A. M. 1988, Sov. Astron. Lett. 14, 284.

Babel, J., and Michaud, G. 1991, A\&A, 248, 155.

Bahcall, J. N., and Loeb, A. 1990, ApJ, 360, 267.

Burgers, J. M. 1969, Flow Equations for Composite Gases, (New York: Academic Press).

Chaboyer, B. and Zahn, J.-P. 1992, A\&A 253, 173.

Chapman, S., and Cowling, T. G. 1970, The Mathematical Theory of non-uniform Gases (3d ed.; Cambridge: Cambridge University Press).

Charbonneau, P., 1992, A\&A, submitted.

Charbonneau, P., 1992, in Cool Stars, Stellar Systems, and the Sun: Seventh Cambridge Workshop, eds. M. S. Giampapa and J. A. Bookbinder, (San Francisco: Astronomical Society of the Pacific), p. 416.

Charbonneau, P., and Michaud, G. 1988, ApJ, 334, 746.

Charbonneau, P., and Michaud, G. 1991, ApJ, 370, 693.

Charbonneau, P., Michaud, G., and Proffitt, C. R. 1989, ApJ, 347, 821.

Charbonnel, C., and Vauclair, S. 1992, A\&A, submitted.

Charbonnel, C., Vauclair, S., and Zahn, J.-P. 1992, A\&A, 255, 191.

Cox, A. N., Morgan, S. M., Rogers, F. J., and Iglesias, C. A. 1992, ApJ, 393, 272. 
Deliyannis, C. P., and Demarque, P. 1991, ApJ, 379, 216.

Deliyannis, C. P., Pinsonneault, H. H., and Duncan, D. K. 1992, ApJ, in press.

Dziembowski, W. A., Pamyatnykh, A. A., and Sienkeiwicz, R. 1992, preprint.

Garcia Lopez, R. J. and Spruit, H. C. 1991, ApJ, 377, 268.

Guzik, J. A., and Cox, A. N. 1992, ApJL., 386, 729.

Kosovichev, A. G., Christensen-Dalsgaard, J., Dappen, W., Dziembowski, W. A., Gough, D. O., and Thompson, M. J. 1992, M.N., in press.

Kurucz, R. L. 1991, in Stellar Atmospheres: Beyond Classical Models, ed. by L. Crivellari, I. Hubeny and D. G. Hummer, NATO ASI Series (Dordrecht, Kluwer), p. 440.

LeBlanc, F., and Michaud, G. 1992, ApJ, submitted.

Martel, A. 1979, Internal Report, Université de Montréal.

Michaud, G. 1976, in Physics of Ap Stars. IAU Colloquium No. 32, ed. W W.

Weiss, H. Jenkner, and H. J. Wood (Universitatsternwarte Wien, Vienna), p.351.

Michaud, G. 1980, AJ, 85, 589.

Michaud, G. 1986, in Upper Main Sequence Stars with Anomalous Abundances, IAU Colloquium 90, Ed. C. R. Cowley, M. M. Dworetsky and C. Mégessier (Doldrecht: Reidel), p. 459.

Michaud, G. 1991a, in IAU Symposium 145, Evolution of Stars: the Photospheric Abundance Connection, Golden Sands, Bulgaria, 25-31 August, ed. G. Michaud and A. Tutukov (Kluwer), pp. 111-124.

Michaud, G. 1991b, Annales de Physique Fr., 16, 481.

Michaud, G., Montmerle, T., Cox, A.N., Magee N.H., Hodson, S.W., and Martel, A. 1979, ApJ, 234, 206.

Michaud, G., and Proffitt, C. R. 1992, in Inside the Stars, IAU COLLOQUIUM 137, Vienna, April 1992, ed. A. Baglin and W. W. Weiss, (Conference Series of the PASP), in press.

Michaud, G., and Richer, J. 1992, in Origin and Evolution of the Elements, Paris, June 1992, ed., N. Prantzos and M. Cassé, (Cambridge University Press), in press. Paquette, C., Pelletier, C., Fontaine, G., and Michaud, G. 1986, ApJS, 61, 177. Pinsonneault, M. H., Deliyannis, C. P., and Demarque, P. 1992, ApJS, 78, 179. Pinsonneault, M. H., Kawaler, S. D., and Demarque, P. 1990, ApJS, 74, 501.

Pinsonneault, M. H., Kawaler, S. D., Sofia, S., and Demarque, P. 1989, ApJ, 338, 424.

Proffitt, C. R., and Michaud, G. 1991a, ApJ, 371, 584.

Proffitt, C. R., and Michaud, G. 1991b, ApJ, 380, 238.

Richer, J. 1992, Thèse de doctorat, Université de Montréal.

Richer, J., and Michaud, G. 1992, in preparation.

Rogers, F. J., and Iglesias, C. A. 1992, ApJS, 79, 507.

Soderblom, D. R., Jones, B. F., Balachandran, S., Stauffer, J. R., Duncan, D. K., Fedele, S., and Hudon, J. D. 1992, AJ, submitted.

Schatzman, E., 1962, Ann. Astrophys 25, 18.

Schatzman, E., 1977, A\&A 56, 211.

Spiegel, E. A. and Zahn, J.-P., 1970, Comments Astrophys. Space Phys. 2, 178.

Spite, F., and Spite, M. 1982, A\&A, 115, 357.

Vauclair, S. 1988, ApJ, 335, 971.

Vauclair, S., Michaud, G., and Charland, Y. 1974, A\&A, 31, 381.

Weber, E.J. and Davis, L. Jr. 1967, ApJ, 148, 217.

Zahn, J.-P. 1992, A\&A, in press.

Zahn, J.-P. 1974, in Stellar Instability and Evolution, ed. P. Ledoux, A. Noels, and A. W. Rodgers (Dordrecht: Reidel), p. 185. 